\title{
Reply to determining structural identifiability of parameter learning machines
}

\author{
D.J. Cole $\mathrm{a}^{\mathrm{a} *}$ \\ ${ }^{a}$ School of Mathematics, Statistics and Actuarial Science, University of Kent, Canterbury, \\ CT2 $7 N F, U K$
}

\begin{abstract}
The paper Ran and Hu (2014, Neurocomputing) examines identifiability and parameter redundancy in classes of models used in machine learning. This note discusses the results on global identifiability and also clarifies that the paper's results on parameter redundancy already exist in the paper Cole et al. (2010, Mathematical Biosciences).

Keywords: derivative matrix, exhaustive summaries, global identifiability, Jacobian matrix, local identifiability, parameter redundancy
\end{abstract}

\section{Introduction}

There is a long history of testing for identifiability of models by testing the rank of a matrix formed by differentiating some representation of a model with respect to the parameters of the models; for example, see $[1,2,3,4]$. However this test only distinguishes whether a model is at least locally identifiable or nonidentifiable, and not whether a model is globally identifiable. The exception is for exponential family models, where the model will either be non-identifiable or globally identifiable [1]. However Ran and $\mathrm{Hu}$ [5] uses a similar test to distinguish between non-identifiable or globally identifiable in cases where the model is not necessarily from the exponential family. In this note we provide

\footnotetext{
* Corresponding author

Email address: d.j.cole@kent.ac.uk (D.J. Cole)

URL: http://www.kent.ac.uk/ims/personal/djc24/index.htm (D.J. Cole)
}

Preprint submitted to Neurocomputing

July 9, 2015 
counter examples which are locally identifiable, but which Ran and $\mathrm{Hu}[5]$ 's theorems would classify as globally identifiable. Maple code for the examples of this note are provided in the supplementary material.

Ran and $\mathrm{Hu}[5]$ also discuss the concept of an exhaustive summary, which is a unique representation of a model. This term is used for continuous state-space models in [3] and then extended to any model with an explicit representation in [6]. One reason a model can be non-identifiable is because it is overparameterised and could be reparameterised in terms of a smaller number of parameters. This is known as parameter redundancy; for example, see $[4,6]$. Section 5 in [5] provides results on parameter redundancy which are already provided in [6].

\section{Parameter Redundancy}

A model, $M(\boldsymbol{\theta})$, with $p$ parameters $\boldsymbol{\theta}=\left[\theta_{1}, \ldots, \theta_{p}\right]$, is parameter redundant if it can be reparameterised as $M(\boldsymbol{\beta})$, with $q$ parameters $\boldsymbol{\beta}=\left[\beta_{1}, \ldots, \beta_{q}\right]$, where $\boldsymbol{\beta}=f(\boldsymbol{\theta})$, for some function $f$ and where $q<p$. Theorem 2a of Cole et al. [6] states that for any exhaustive summary of a model, $\boldsymbol{\kappa}$, it can be determined whether or not a model is parameter redundant by calculating the rank of the derivative matrix $\mathbf{D}=\left[\partial \kappa_{j} / \partial \theta_{i}\right]$. If the rank is less than $p$ then the model is parameter redundant. This is identical to Theorem 6 of [5] but published four years earlier. Theorem $2 \mathrm{~b}$ of $[6]$ generalises results from $[7,8,9]$ to allow for a reparameterisation that results in a model that is no longer parameter redundant. Such a reparameterisation is known as locally identifiable reparameterisations $[8,9]$ or estimable parameter combinations [7].

The rank of the derivative matrix can be found using a symbolic algebra package such as Maple. In this paper, Maple version 18 was used. Maple can theoretically be used to check for parameter redundancy in any model, with an explicit exhaustive summary. However in structurally complex models this becomes computationally infeasible. Examples are given in Cole et al. [6]. This problem can be solved by using a structurally simpler exhaustive summary which can be seen in example 7 and 8 of [5]. Further results in Cole 
et al. [6] provide a framework for finding simpler exhaustive summaries using reparameterisation. Primarily the use of this framework has been in ecological models, see for example $[10,11]$, however [6] also demonstrates the use of this method in compartment models.

\section{Local and Global Identifiability}

A model, $M(\boldsymbol{\theta})$, is globally identifiable if $M\left(\boldsymbol{\theta}_{1}\right)=M\left(\boldsymbol{\theta}_{2}\right)$ implies that $\boldsymbol{\theta}_{1}=\boldsymbol{\theta}_{2}$, is locally identifiable if there exists an open neighbourhood of any $\boldsymbol{\theta}$ such that this is true. Otherwise $M(\boldsymbol{\theta})$ is non-identifiable. A parameter redundant model will be non-identifiable $[4,6]$.

The first class of models examined in [5] are Multiple-Input Multiple-Output (MIMO) models, which are defined by $y_{i}=f_{i}(\mathbf{x}, \boldsymbol{\theta})$, where $\mathbf{x}=\left[x_{1}, \ldots, x_{n}\right]$ is the input vector of length $n, \mathbf{y}=\left[y_{1}, \ldots, y_{m}\right]$ is the output vector of length $m$ and $\boldsymbol{\theta}=\left[\theta_{1}, \ldots, \theta_{k}\right]$ is a vector of $k$ parameters. Theorem 1 of [5] states that the MIMO model is globally identifiable if and only if the partial derivative matrix $\mathbf{D}=\left[\partial f_{i} / \partial \theta_{j}\right]$ is of full column rank.

Consider an example where $y_{1}=a^{2} x_{1}+b^{2} x_{2}$ and $y_{2}=a x_{1}$, with parameters $\boldsymbol{\theta}=[a, b]$. If $a=1$ and $b=2$ then the output is $y_{1}=x_{1}+4 x_{2}$ and $y_{2}=x_{1}$. If $a=1$ and $b=-2$ then the output is identical. As two different values of the parameter $b$ give the same output the model is locally identifiable unless the parameter space is restricted. Using Theorem 1 of [5] the partial derivative matrix

$$
\mathbf{D}=\left[\frac{\partial f_{i}}{\partial \theta_{j}}\right]=\left[\begin{array}{cc}
2 a x_{1} & 2 b x_{2} \\
x_{1} & 0
\end{array}\right]
$$

is of full column rank 2, which leads to the incorrect conclusion that the model is globally identifiable. Using the methods of [6] with exhaustive summary $\boldsymbol{\kappa}=\left[a^{2} x_{1}+b^{2} x_{2}, a x_{1}\right]^{T}$ the resulting derivative matrix is the transpose of (1). However this is only used to distinguish whether or not a model is nonidentifiable. Theorem 9 of [6] gives a test for global identifiability by solving the set of equations $\kappa_{i}=\eta_{i}$. A unique solution indicates the model is globally 
identifiable, otherwise a model with a full rank derivative matrix would be locally identifiable. There is a unique solution for $a$ of $\eta_{2} / x_{1}$ however there are two solutions for $b$ of $\pm \sqrt{\left(\eta_{1} x_{1}-\eta_{2}^{2}\right) /\left(x_{1} x_{2}\right)}$, unless $\eta_{1} x_{1}=\eta_{2}^{2}$. Except when $\eta_{1} x_{1}=\eta_{2}^{2}$, the model is not globally identifiable and is only locally identifiable.

Theorem 4 of [5] tests identifiability of a stochastic model, using an exhaustive summary, $\mathbf{s}(\boldsymbol{\theta})$. If the Jacobian matrix $\mathbf{J}=\partial \mathbf{s} / \partial \boldsymbol{\theta}$ is full rank, then under certain conditions the model is globally identifiable. Example 4 of [5] considers a second-order state-space model with

$$
\left[\begin{array}{l}
x_{1}(t+1) \\
x_{2}(t+1)
\end{array}\right]=\left[\begin{array}{cc}
\theta_{1} & 0 \\
1 & \theta_{2}
\end{array}\right]\left[\begin{array}{l}
x_{1}(t) \\
x_{2}(t)
\end{array}\right], y(t)=x_{2}(t), x_{1}(0)=x_{2}(0)=0,
$$

where the parameters are $\boldsymbol{\theta}=\left[\theta_{1}, \theta_{2}\right]$ and $\epsilon(t)$ follows a Gaussian distribution with mean 0 and variance 1 . Theorem 4 of [5] is used to show the model is globally identifiable. However this model is in fact locally identifiable. Consider the output given by equation (57) of [5], which is

$$
\left[\begin{array}{l}
y(1) \\
y(2) \\
y(3) \\
y(4)
\end{array}\right]=\left[\begin{array}{c}
0 \\
\epsilon(0) \\
\left(\theta_{1}+\theta_{2}\right) \epsilon(0)+\epsilon(1) \\
\left(\theta_{1}^{2}+\theta_{1} \theta_{2}+\theta_{2}^{2}\right) \epsilon(0)+\left(\theta_{1}+\theta_{2}\right) \epsilon(1)+\epsilon(2)
\end{array}\right] .
$$

If $\theta_{1}=0$ and $\theta_{2}=2$ or $\theta_{1}=2$ and $\theta_{2}=0$ then $y(3)=2 \epsilon(0)+\epsilon(1)$ and $y(4)=4 \epsilon(0)+2 \epsilon(1)+\epsilon(2)$. As there are two parameter values that give identical output this model is locally not globally identifiable. To use the methods of [6] for detecting global identifiability we first find a simpler exhaustive summary. The original exhaustive summary used in [5] is

$$
\boldsymbol{\kappa}(\boldsymbol{\theta})=\left[\begin{array}{c}
\theta_{1}+\theta_{2} \\
\theta_{1}^{2}+\theta_{1} \theta_{2}+\theta_{2}^{2} \\
\left(\theta_{1}+\theta_{2}\right)^{2}+1 \\
\left(\theta_{1}+\theta_{2}\right)\left(\theta_{1}^{2}+\theta_{1} \theta_{2}+\theta_{2}^{2}+1\right) \\
\left(\theta_{1}^{2}+\theta_{1} \theta_{2}+\theta_{2}^{2}\right)^{2}+\left(\theta_{1}+\theta_{2}\right)^{2}+1
\end{array}\right],
$$

with parameters $\boldsymbol{\theta}=\left[\theta_{1}, \theta_{2}\right]$. A reparameterisation is $\mathbf{s}=\left[s_{1}, s_{2}\right]^{T}=\left[\theta_{1}+\right.$ $\left.\theta_{2}, \theta_{1}^{2}+\theta_{1} \theta_{2}+\theta_{2}^{2}\right]^{T}$. The original exhaustive summary can be rewritten as 
$\boldsymbol{\kappa}(\mathbf{s})=\left[s_{1}, s_{2}, s_{1}^{2}+1, s_{1}\left(s_{2}+1\right), s_{1}^{2}+s_{2}^{2}+1\right]^{T}$. The derivative matrix

$$
\frac{\partial \boldsymbol{\kappa}(\mathbf{s})}{\partial \mathbf{s}}=\left[\begin{array}{ccccc}
1 & 0 & 2 s_{1} & s_{2}+1 & 2 s_{1} \\
0 & 1 & 0 & s_{1} & 2 s_{2}
\end{array}\right]
$$

is of full rank 2, therefore by Theorem 8 of [6] $\mathbf{s}$ is an exhaustive summary and the model is at least locally identifiable. However there are two solution to the equation $\mathbf{s}=\boldsymbol{\eta}, \theta_{1}=\eta_{1} / 2-\sqrt{4 \eta_{2}-3 \eta_{1}^{2}} / 2, \theta_{2}=\eta_{1} / 2+\sqrt{4 \eta_{2}-3 \eta_{1}^{2}} / 2$ and $\theta_{1}=\eta_{1} / 2+\sqrt{4 \eta_{2}-3 \eta_{1}^{2}} / 2, \theta_{2}=\eta_{1} / 2-\sqrt{4 \eta_{2}-3 \eta_{1}^{2}} / 2$, except when $4 \eta_{2}=3 \eta_{1}^{2}$. By Theorem 9 of [6], this model is only locally identifiable, if $4 \eta_{2} \neq 3 \eta_{1}^{2}$.

\section{Discussion}

Ran and $\mathrm{Hu}[5]$ provide theorems for checking whether a model is locally identifiability in models used in machine learning. Global identifiability cannot generally be checked by calculating the rank of an appropriate Jacobian or derivative matrix.

Acknowledgements The author would like to thank Dr Neil Evans and Prof Byron Morgan for helpful discussions of this paper.

[1] T. J. Rothenberg, Identification in parametric models, Econometrica 39 (1971) 577-591.

[2] L. A. Goodman, Exploratory latent structure analysis using both identifiable and unidentifiable models, Biometrika 61 (1974) 215-231.

[3] E. Walter, Y. Lecourtier, Global approaches to identifiability testing for linear and nonlinear state space models, Mathematics and Computers in Simulation 24 (1982) 472-482.

[4] E. A. Catchpole, B. J. T. Morgan, Detecting parameter redundancy, Biometrika 84 (1997) 187-196.

[5] Z.-Y. Ran, B.-G. Hu, Determining structural identifiability of parameter learning machines, Neurocomputing 127 (2014) 88-97. 
[6] D. J. Cole, B. J. T. Morgan, D. M. Titterington, Determining the parametric structure of models, Mathematical Biosciences 228 (2010) 16-30.

[7] E. A. Catchpole, B. J. T. Morgan, S. N. Freeman, Estimation in parameter redundant models, Biometrika 85 (1998) 462-468.

[8] M. J. Chappell, R. N. Gunn, A procedure for generating locally identifiable reparameterisations of unidentifiable non-linear systems by the similarity transformation approach, Mathematical Biosciences 148 (1998) 21-41.

[9] N. D. Evans, M.J. Chappell, Extensions to a procedure for generating locally identifiable reparameterisations of unidentifiable systems, Mathematical Biosciences 168 (2000) 137-159.

[10] D. J. Cole, Determining parameter redundancy of multi-state markrecapture models for sea birds, Journal of Ornithology 152 (2012) S305S315.

[11] D. J. Cole, B. J. T. Morgan, R. S. McCrea, R. Pradel, O, Gimenez, R. Choquet, Does your species have memory? Capture-Recapture Data with Memory Models, Ecology and Evolution 4 (2014) 2124-2133. 